\title{
ПСИХОФАРМАКОТЕРАПИЯ
}

\author{
УДК 631.524.824:004.81:616.89-008.441.13:616-039.34
}

Для цитирования: Максимова И.В., Березовская М.А. Динамика когнитивных расстройств при включении кортексина в алгоритм терапии пациентов с алкогольной зависимостью, перенесших генерализованный тонико-клонический приступ на момент госпитализации. Сибирский вестник психиатрии и наркологии. 2021; 2 (111): 82-87. https://doi.org/10.26617/1810-3111-2021-2(111)-82-87

\section{Динамика когнитивных расстройств при включении кортексина в алгоритм терапии пациентов с алкогольной зависимостью, перенесших генерализованный тонико- клонический приступ на момент госпитализации}

\section{Максимова И.В., Березовская М.А.}

ФГБОУ ВО «Красноярский государственный медицинский университет им. проф. В.Ф. Войно-Ясенецчкого» Минздрава России

Россия, 660022, Красноярск, ул. Партизана Железняка, 1

\section{PEЗЮME}

Актуальность. Описаны эффекты кортексина при синдроме хронической усталости, травматической болезни головного мозга и черепно-мозговой травме, резистентной параноидной шизофрении, психосоматических нарушениях у ликвидаторов радиационных аварий, разных видах инсультов. Оптимизация процесса лечения с использованием кортексина способствует восстановлению и стабилизации высших мозговых, когнитивных и двигательных функций, активизации обмена дофамина. Цель исследования: изучение динамики когнитивных расстройств при включении кортексина в схему лечения пациентов с алкогольной зависимостью, перенесших генерализованный тонико-клонический приступ на момент госпитализации. Материалы и методы: в исследование включены 40 пациентов в возрасте от 23 до 60 лет с диагностированной по МКБ-10 алкогольной зависимостью (F10.2), перенесших генерализованный тонико-клонический приступ (G40.6), рандомизированные в две группы. Основная группа состояла из 20 человек, всем им был назначен кортексин в дозе 10 мг в течение 10 дней. Группа сравнения включала 20 человек, получавших стандартное лечение (дезинтоксикационная и ноотропная терапия, витаминотерапия). Для оценки когнитивных расстройств до и после лечения использовалась Краткая шкала психического статуса (MMSE). Результаты. Введение в схему лечения когнитивных расстройств при алкогольной зависимости кортексина, оказывающего нейропротекторное действие, улучшает когнитивные функции: средний балл по шкале MMSE до и после лечения кортексином статистически значимо $(\mathrm{p}<0,05)$ возрастает у пациентов основной группы - c $25 \pm 2,64$ до $29 \pm 0,48$, в группе сравнения - с $26 \pm 1,18$ до $27 \pm 2,35$.

Ключевые слова: алкогольная зависимость, генерализованный тонико-клонический приступ, когнитивные нарушения, оценка эффективности кортексина.

\section{ВВЕДЕНИЕ}

Одной из наиболее распространенных форм хронического поражения головного мозга при алкогольной зависимости является энцефалопатия, клинически реализующаяся различными вариантами неврологических проявлений $[1,2,3,4$, $5,6,7]$. Понятие «алкогольная энцефалопатия» включает в себя комплекс неврологических и нейропсихологических нарушений, патопсихологических симптомокомплексов, возникающих как результат системного поражения вследствие токсического действия алкоголя на головной мозг. Одним из ведущих синдромов алкогольного поражения головного мозга является прогрессирующее снижение когнитивных функций $[8,9,10$, $11,12,13,14]$.
По данным различных авторов, эти нарушения обнаруживаются в 50-70\% случаев, причем в 10\% случаев они носят выраженный характер, достигая степени деменции $[15,16,17,18,19,20]$. Наличие генерализованных тонико-клонических приступов в клинике алкогольной зависимости может вызывать прогрессирование когнитивных нарушений $[21,22,23,24,25]$. Становится очевидным, что выявление когнитивных нарушений и их лечение при алкогольной зависимости является актуальным вопросом современной психиатрии и наркологии. В настоящее время при лечении когнитивных расстройств, возникших на фоне алкогольной зависимости, согласно стандартам, утвержденным МЗ РФ, применяются витамины группы В и ноотропные препараты. 
Кортексин - многокомпонентный препарат, получаемый из коры головного мозга скота, представляет собой комплекс низкомолекулярных водорастворимых полипептидных фракций, непосредственно проникающих через гематоэнцефалический барьер к нервным клеткам. Обладает тканеспецифическим многофункциональным действием на головной мозг, что проявляется в метаболической регуляции, нейропротекции, функциональной нейромодуляции, нейротрофической активности. Помимо того, повышает эффективность энергетического метаболизма клеток мозга, улучшает внутриклеточный синтез белка, оказывает антистрессорный эффект. Одновременно регулирует процессы перекисного окисления липидов в нейронах головного мозга, снижает образование свободных радикалов, блокирует процессы окисления. При его использовании в процессе лечения устраняется дисбаланс тормозных и возбуждающих аминокислот, отмечается положительное действие при нарушении когнитивных функций, улучшаются концентрация внимания, кратковременная память, наблюдается улучшение эмоционально-мотивационной сферы, повышается способность к обучению, ускоряется восстановление функций головного мозга после стрессорных воздействий, регулируются уровни серотонина и дофамина. Кортексин стимулирует процесс физической и умственной деятельности, не вызывая избыточного активирующего влияния, восстанавливает биоэлектрическую активность головного мозга, улучшает репаративные процессы в головном мозге. При его применении отсутствуют побочные эффекты. Благодаря церебропротекторному, ноотропному, вазоактивному, нейротропному действию и стимулирующему эффекту на психические функции кортексин успешно применяется в терапии болезней зависимости, приводит к снижению невротизации, тревоги, беспокойства, напряженности и раздражительности, улучшает самочувствие и настроение пациентов, повышает чувство удовлетворенности жизнью и позволяет достичь максимальной психосоциальной реабилитации. Всё это представляет интерес в лечении пациентов с алкогольной зависимостью, перенесщих генерализованный тонико-клонический приступ [2].

\section{ЦЕЛЬ ИССЛЕДОВАНИЯ}

Сравнительно-клиническое исследование динамики когнитивных расстройств при включении в стандартную схему лечения нейропротектора кортексина у пациентов с алкогольной зависимостью, перенесших генерализованный тоникоклонический приступ на момент госпитализации, и у пациентов, получавших только дезинтоксикационную и ноотропную терапию, витаминотерапию.

\section{МАТЕРИАЛЫ И МЕТОДЫ}

В общую исследовательскую выборку включено 40 пациентов мужского пола в возрасте от 23 до 60 лет (средний возраст составил 30,5 $\pm 10,7$ года), страдающих алкогольной зависимостью II стадии (F10.242). Все они находились на стационарном лечении в Красноярском краевом наркологическом диспансере № 1 в период с 2014 по 2016 г. и перенесли либо синдром отмены алкоголя с судорогами (F10.31), либо алкогольный делирий с судорогами (F10.41).

Критерии невключения: возраст до 18 лет, наличие другого психическое заболевания (в том числе острое психотическое состояние), нежелание больного соблюдать протокол настоящего исследования, наркотическая или другая аддиктивная зависимость, наличие эпилепсии в анамнезе. Все пациенты дали добровольное информированное согласие на выполнение протокола исследования.

Пациенты были рандомизированы в две группы. К основной группе отнесены 20 человек (средний возраст составил 30,7 $\pm 11,9$ года), с 4-го дня пребывания в стационаре они получали кортексин в течение 10 дней, внутримышечно, в дозе 10 мг. В группу сравнения вошло 20 человек, средний возраст которых составил 29,4 1 17,3 года; для коррекции когнитивных нарушений использовалась стандартная симптоматическая базисная терапия ноотропными препаратами (пирацетам, винпоцетин, глицин).

Оценка когнитивных функций проводилась в соответствии с данными обследования с помощью методики Краткая шкала психического статуса (MMSE). В динамике оценивалась эффективность кортексина в двух точках исследования: до и после лечения. Клинические проявления оценивались с учетом субъективных основных ощущений в плане редукции соматической, неврологической, психопатологической симптоматики абстинентного синдрома.

Статистическая обработка полученных данных выполнялась при помощи программы SPSS (версия 22.0). Описательная статистика результатов исследования представлена в виде средних арифметических (М) и стандартных отклонений (б). Значения средних арифметических имеют вид $\mathrm{M} \pm \sigma$. При отсутствии нормального распределения переменных в описательной статистике использовались медиана (Ме) и перцентили (Р25, Р75). Значения средних величин отображались в этом случае как Ме (P25; Р75). Оценка возможных сдвигов при неврологических и когнитивных нарушениях осуществлялась с применением tтеста для определения значений зависимых и независимых переменных. 


\section{РЕЗУЛЬТАТЫ И ОБСУЖДЕНИЕ}

Пациенты обеих исследовательских групп были сопоставимы по возрасту, уровню образования, длительности заболевания, выраженности когнитивных нарушений, сопутствующим заболеваниям, неврологической симптоматике. За время пребывания в наркологическом стационаре у пациентов обеих групп не отмечалось повторных генерализованных тонико-клонических приступов.

При объективном исследовании неврологического статуса (высшие корковые, двигательные и вегетативные функции, чувствительность) у пациентов преобладала разнообразная микроочаговая рассеянная симптоматика: установочный горизонтальный нистагм в крайних отведениях глазных яблок - у 11 (27,5\%) пациентов, изменения рефлекторного фона - у 24 (60\%), двухсторонняя легкая мозжечковая недостаточность (атаксия) - более чем у половины исследуемых $(\mathrm{n}=28,70,0 \%)$, редкие чувствительные нарушения с доминированием в нижних конечностях в виде гипестезии и гиперестезии, которые преимущественно выявлялись на фоне сниженного рефлекторного фона - у 11 (27,5\%). Вместе с тем двигательные расстройства (атаксия), симптомы орального автоматизма, сгибательные/разгибательные патологические стопные и кистевые рефлексы не были зафиксированы. Выявленная микроочаговая неврологическая симптоматика (двигательная, координаторная, зрительная) свидетельствует о токсическом и гипоксическом повреждении/поражении ЦНС, с чем и связана клиническая картина когнитивных нарушений с характерным прогрессирующим нарастанием.

Оценка когнитивных нарушений с помощью методики Краткая шкала психического статуса (MMSE) осуществлялась на 4-й день нахождения пациентов в стационаре, когда были купированы выраженные вегетативные, соматовегетативные и неврологические проявления абстинентного синдрома. Состояние пациентов после проведенного стационарного лечения кортексином и по стандартному протоколу оценивалось в динамике. В сравниваемых группах было выявлено следующее: до лечения в основной группе пациентов, получавших кортексин, средний балл, составлявший 25 (min-max 18-26), был ниже среднего балла в группе сравнения - 26 (min-max 18-27). После проведения двух видов терапии (с кортексином и стандартной симптоматической базисной ноотропной терапии) отмечалось повышение суммарного показателя в баллах по нейропсихологической методике MMSE: до 29 (min-max 25-30) в основной группе (подъем показателя до отсутствия когнитивных нарушений) и до 27 (min-max 22-30) - в группе сравнения (на уровне преддементных нарушений). Обсуждаемые данные приведены в таблице 1.

Т а б л и ц а 1. Сравнительная оценка динамики когнитивных нарушений у больных алкогольной зависимостью в процессе лечения с включением кортексина и по стандартной схеме терапии

\begin{tabular}{|l|c|c|c|c|}
\hline \multirow{2}{*}{\multicolumn{1}{|c|}{ Показатель }} & \multicolumn{2}{|c|}{ Основная группа $(\mathrm{n}=20)$} & \multicolumn{2}{c|}{ Группа сравнения $(\mathrm{n}=20)$} \\
\cline { 2 - 5 } & До лечения & После лечения & До лечения & После лечения \\
\hline Средний балл по шкале MMSE & $25 \pm 2,64$ & $29 \pm 0,48(\mathrm{p} \leq 0,05)$ & $26 \pm 1,18$ & $27 \pm 2,35$ \\
\hline P25; Р75 & $23 ; 26$ & $27 ; 30$ & $26 ; 29$ & $25 ; 28$ \\
\hline Минимальное значение & 18 & 25 & 18 & 22 \\
\hline Максимальное значение & 26 & 30 & 27 & 30 \\
\hline
\end{tabular}

По результатам собственного исследования кортексин показал высокую терапевтическую эффективность, безопасность, хорошую переносимость и отсутствие побочных токсических и аллергических реакций. Следует отметить, что на фоне терапии кортексином не зарегистрировано клинически значимой динамики показателей жизненно важных функций организма, соматического или неврологического статуса. Удобство применения кортексина состояло в краткосрочном курсе на протяжении 10 дней, что позволяет применять препарат в условиях наркологического стационара.

Ранее в литературе описывались случаи лечения кортексином когнитивных нарушений при инсультах, травматическом поражении головного мозга и продемонстрировано его влияние на про- цессы апоптоза у больных алкоголизмом. При этом клинических исследований о влиянии кортексина на купирование алкогольного абстинентного синдрома и улучшение когнитивного функционирования не проводилось.

По итогам сравнительного клиникокатамнестического наблюдения пациентов основной группы и группы сравнения на амбулаторном этапе обнаружены статистически значимые $(\mathrm{p} \leq 0,05)$ различия по темпу прогредиентности алкоголизма. Случаи срыва ремиссии в виде повторных алкогольных эксцессов и необходимость повторных госпитализаций после выписки в течение периода катамнестического наблюдения в основной группе регистрировались с более редкой частотой $(\mathrm{n}=4,20 \%)$ при сопоставлении с группой сравнения $(\mathrm{n}=15,75 \%)$. 


\section{ЗАКЛЮЧЕНИЕ}

Включение в терапевтический алгоритм пациентов с когнитивными нарушениями при алкогольной зависимости, перенесших генерализованный тонико-клонический приступ, кортексина, оказывающего нейопротекторное, ноотропное, антиоксидантное и противосудорожное действие, приводит к существенной редукции когнитивных расстройств. Это подтверждается более выраженным повышением суммарного показателя в баллах по шкале MMSE в основной группе - до 29 (min-max 25-30), чем в группе сравнения - до 27 (min-max 22-30) на фоне стандартной терапии. Кортексин оказывает выраженный клинический эффект: улучшение когнитивных функций, общего фона настроения, мотивации и инициативы, повышение скорости реагирования, концентрации внимания и умственной работоспособности.

\section{КОНФЛИКТ ИНТЕРЕСОВ}

Авторы заявляют об отсутствии явных и потенциальных конфликтов интересов в связи с публикацией данной статьи.

\section{ИСТОЧНИК ФИНАНСИРОВАНИЯ}

Работа выполнена по целевым комплексным программам основного плана НИР ГБОУ ВПО «Красноярский государственный медицинский университет» и не имела спонсорского или иного финансирования при проведении исследования.

\section{СООТВЕТСТВИЕ ПРИНЦИПАМ ЭТИКИ}

Работа соответствует этическим стандартам Хельсинской декларации ВМА (протокол заседания этического комитета ГБОУ ВПО «Красноярский государственный медицинский университет им. проф. В.Ф. Войно-Ясенецкого» Минздрава России № 59/2014 от 02 декабря 2014 г.).

\section{ЛИТЕРАТУРА/REFERENCES}

1. Андрианова Е.Д., Дамулин И.В., Сиволап Ю.П. Когнитивные расстройства при алкоголизме. Наркология. 2013. Т. 12, № 138. С. 79-85. Andrianova ED, Damulin IV, Sivolap YuP. Cognitive disorders in alcoholism. Narcology. 2013;12 (138):79-85 (in Russian).

2. Бохан Н.А., Иванова С.А., Мандель А.И., Жернова Е.В., Кисель Н.И. Когнитивные функции и процессы апоптоза у больных алкоголизмом: эффекты нейрометаболической коррекции. Наркология. 2012. Т. 11, № 7 (127). С. 51-55. Bokhan NA, Ivanova SA, Mandel AI, Zhernova EV, Kisel NI. Cognitive functions and processes of apoptosis in patients with alcoholism: the effects of neurometabolic correction. Narcology. 2012;11,7(127):51-55 (in Russian).

3. Зиновьева О.Е., Емельянова А.Ю. Неврологические проявления алкогольной болезни: роль витаминов группы В в лечении. Consilium Medicum. 2016. T. 18, № 9). C. 133-138. Zinovieva OE, Emelyanova $\mathrm{AYu}$. Neurological manifestations of alcoholic disease: the role of vitamin $\mathrm{B}$ in the treatment. Consilium Medicum. 2016;18(9):133138 (in Russian).

4. Казенных Т.В. Методологические подходы к реабилитации больных пароксизмальными состояниями. Сибирский вестник психиатрии и наркологии. 2014. № 2 (83). С. 74-77. Kazennykh TV. Methodological approaches to rehabilitation of patients with paroxysmal states. Siberian Herald of Psychiatry and Addiction Psychiatry.2014;2(83):74-77 (in Russian).

5. Катаманова Е.В., Рукавишников В.С., Лахман О.Л., Шевченко О.И., Денисова И.А. Когнитивные нарушения при токсическом поражении мозга. Журнал неврологии и психиатрии им. С.С. Корсакова. 2015. Т. 115, № 2. С. 11-15. Katamanova EV, Rukavishnikov VS, Lakhman OL, Shevchenko OI, Denisova IA. Cognitive impairment in toxic brain damage. S.S. Korsakov Journal of Neurology and Psychiatry. 2015;115(2):11-15. 2015; $115 \quad$ (2): $11-15 \quad$ (in Russian). DOI 10.17116/jnevro20151152111-15

6. Зарва В.А., Осенкова К.Е. Влияние употребления алкоголя на состояние зрительнопространственных функций в ранней взрослости. Молодежный научный вестник. 2018. № 1 (26). C. 61-65. Zarva VA, Osenkova KE. The influence of alcohol consumption on the state of visual-spatial functions in early adulthood. Youth Scientific Bulletin. 2018;1(26):61-65 (in Russian).

7. Захаров В.В. Злоупотребление алкоголем: неврологические осложнения и современные подходы к терапии. Эффективная фармакотерапия. 2014. № 8. С. 24. Zakharov VV. Alcohol abuse: neurological complications and current therapeutic approaches. Effective Pharmacotherapy. 2014;8:24 (in Russian).

8. Bernardin F, Maheut-Bosser A, Paille F. Troubles cognitifs du sujet alcoolodépendant [Cognitive impairment of alcohol-dependent subjects]. Rev Prat. 2014 Apr;64(4):462-5. French. PMID: 24855773.

9. Campagna F, Montagnese S, Schiff S, Ruzzoli M, Biancardi A, Iannizzi P, Pujatti PL, Angeli P, Gatta A, Merkel C, Leandro G, Mapelli D, Amodio P. Confounders in the detection of minimal hepatic encephalopathy: a neuropsychological and quantified EEG study. Liver Int. 2015 May;35(5):152432. doi: 10.1111/liv.12635. Epub 2014 Jul 23. PMID: 25040245.

10. Бохан Н.А., Приленский Б.Ю., Асаинов Р.Р., Бухна А.Г. Особенность клинической картины и психологического статуса лиц, имеющих соматические осложнения от злоупотребления алкоголя в период длительной ремиссии. Академический журнал Западной Сибири. 2016. Т. 12, № 6 (67). C. 32-35. Bokhan NA, Prilensky BYu, Asainov RR, Bukhna AG. The features of the clinical picture and psychological status of people who have medical complications of alcohol abuse during long-term remission. Academic Journal of Western Siberia. 2016;12,6(67):32-35 (in Russian). 
11. Каменева Н.Н., Куташов В.А. Современный подход к лечению токсической алкогольной энцефалопатии. Молодой ученый. 2015. № 19 (99). C. 274-277. Kameneva NN, Kutashov VA. Modern approach to the treatment of toxic alcoholic encephalopathy. Young Scientist. 2015;19(99):274277 (in Russian).

12. Кушнир Г.М., Иошина Н.Н. Эпилептические приступы у больных алкоголизмом. Таврический журнал психиатрии. 2015. Т. 19, № 2 (71). C. 10-13. Kushnir GM, Ioshina NN. Epileptic seizures in patients with alcoholism. Tavrichesky Journal of Psychiatry. 2015;19,2(71):10-13 (in Russian).

13. Мандель А.И., Бохан Н.А., Шушпанова Т.В., Кисель Н.И. Перспективы применения антиконвульсантов в терапии осложненных форм алкоголизма. Академический журнал Западной Сибири. 2014. Т. 10, № 3 (52). С. 65-66. Mandel AI, Bokhan NA, Shushpanova TV, Kisel NI. Prospects for the application of anticonvulsants in the treatment of complicated forms of alcoholism. Academic Journal of Western Siberia. 2014;10,3(52):65-66 (in Russian).

14. Новохацки А.В., Денисламов М.М. Клиникопсихологические особенности органических расстройств при хроническом алкоголизме. Вестник Южно-Уральского государственного университета. Серия: Психология. 2015. Т. 8, № 4. C. 61-69. Novokhatski AV, Denislamov MM. Clinical and psychological characteristics of organic disorders in chronic alcoholism. Bulletin of South Ural State University. Series: Psychology. 2015;8(4):61-69 (in Russian). DOI: $10.14529 / \mathrm{psy} 150407$

15. Полякова О.В., Юсупова О.Л. Нарушения памяти при алкоголизме. Новая наука: Проблемы и перспективы. 2017. Т. 2, № 3. С. 19-21. Polyakova OV, Yusupova OL. Memory impairment in alcoholism. New Science: Problems and Prospects. 2017;2(3):19-2 (in Russian).

16. Gray JC, MacKillop J. Using Behavior Economics to Understand Alcohol Use Disorders: A Concise Review and Identification of Research Priorities. Curr Addict Rep. 2015 Mar;2(1):68-75. doi: 10.1007/s40429-015-0045-z. Epub 2015 Jan 27. PMID: 27795939; PMCID: PMC5084851.

17. Welch KA. Alcohol consumption and brain health. BMJ. 2017 Jun 6;357:j2645. doi: 10.1136/bmj.j2645. PMID: 28588039; PMCID: PMC5460585.

18. WHO. Global status report on alcohol and health. Geneva: World Health Organization, 2014.

19. Friedrich MJ. New Figures on Global Dementia Cases. doi:10.1001/jama.2015.13039

20. Kim TK, Jung ES, Park JM, Kang K, Lee WW, Lee JJ. An Atypical Presentation of Subacute Encephalopathy with Seizures in Chronic Alcoholism Syndrome. J Epilepsy Res. 2016;6(1):28-30. Published 2016 Jun 30. doi:10.14581/jer.16005

21. Liang J, Olsen RW. Alcohol use disorders and current pharmacological therapies: the role of GABA(A) receptors. Acta Pharmacol Sin. 2014 Aug;35(8):981-93. doi: 10.1038/aps.2014.50. PMID: 25066321; PMCID: PMC4125717.

22. Higuchi S, Matsushita S, Hasegawa Y, Muramatsu $\mathrm{T}$, Arai H. Pupillary response to tropicamide in Japanese patients with alcoholic dementia, Alzheimer's disease, and vascular dementia. Exp Neurol. 1997 Mar;144(1):199-201. doi: 10.1006/exnr.1997.6417. PMID: 9126171.

23. Perry CJ. Cognitive Decline and Recovery in Alcohol Abuse. J Mol Neurosci. 2016 Nov;60(3):383389. doi: 10.1007/s12031-016-0798-4. Epub 2016 Jul 27. PMID: 27460131.

24. Sachdeva A, Chandra M, Choudhary M, Dayal P, Anand KS. Alcohol-Related Dementia and Neurocognitive Impairment: A Review Study. Int J High Risk Behav Addict. 2016 Feb 7;5(3):e27976. doi: 10.5812/ijhrba.27976. PMID: 27818965; PMCID: PMC5086415.

25. Welch KA. Alcohol consumption and brain health. BMJ. 2017 Jun 6;357:j2645. doi: 10.1136/bmj.j2645. PMID: 28588039; PMCID: PMC5460585.

Поступила в редакцию 1.03.2021

Утверждена к печати 28.05.2021

Максимова Ирина Викторовна - к.м.н., доцент кафедры психиатрии, наркологии с курсом ПО. AuthorID РИНЦ 771740. ORСID iD 0000-0001-5076-5368. ResearcherID M-5458-2014. zabigylina@ mail.ru

Березовская Марина Альбертовна - д.м.н., заведующий кафедрой психиатрии, наркологии с курсом ПО. Author ID 620131.mberezovska@mail.ru

Березовская Марина Альбертовна, mberezovska@mail.ru 
UDC 631.524.824:004.81:616.89-008.441.13:616-039.34

For citation: Maksimova I.V., Berezovskaya M.A. Dynamics of cognitive impairments when cortexin is included in the therapy algorithm for patients with alcohol dependence who have undergone a generalized tonic-clonic seizure at the time of hospitalization. Siberian Herald of Psychiatry and Addiction Psychiatry. 2021; 2 (111): 82-87. https://doi.org/10.26617/1810-3111-2021-2(111)-82-87

\title{
Dynamics of cognitive impairments when cortexin is included in the therapy algorithm for patients with alcohol dependence who have undergone a generalized tonic-clonic seizure at the time of hospitalization
}

\section{Maksimova I.V., Berezovskaya M.A.}

Krasnoyarsk State Medical University named after Prof. V.F. Voino-Yasenetsky

Partizan Zheleznyak Street 1, 660022, Krasnoyarsk, Russian Federation

\begin{abstract}
Background. The effects of cortexin in chronic fatigue syndrome, traumatic brain disease and traumatic brain injury, resistant paranoid schizophrenia, psychosomatic disorders in liquidators of radiation accidents, and various types of strokes are described. Optimization of the treatment process with the use of cortexin contributes to the recovery and stabilization of higher cerebral, cognitive and motor functions, activation of dopamine metabolism. Objective: to study the dynamics of cognitive disorders when cortexin is included in the treatment regimen for patients with alcohol dependence who have undergone a generalized tonic-clonic seizure at the time of hospitalization. Materials and Methods: the study included 40 patients aged 23 to 60 years, with alcohol dependence diagnosed according to ICD10 (F10.2), who had a generalized tonic-clonic seizure (G40.6), randomized into two groups. The main group consisted of 20 individuals, all of them were prescribed Cortexin at a dose of $10 \mathrm{mg}$ for 10 days. The comparison group included 20 individuals who received standard treatment (detoxification and nootropic therapy, vitamin therapy). The Mini-Mental Status Scale (MMSE) was used to assess cognitive impairment before and after treatment. Results. The introduction of Cortexin with an neuroprotective effect, into the treatment regimen for cognitive disorders in alcohol dependence, improves cognitive functions: the mean MMSE score before and after treatment with Cortexin statistically significantly $(\mathrm{p}<0.05)$ increases in patients of the main group -from $25 \pm 2.64$ to $29 \pm 0.48$, in the comparison group - from $26 \pm 1.18$ to $27 \pm 2.35$.
\end{abstract}

Keywords: alcohol dependence, generalized tonic-clonic seizure, cognitive impairment, assessment of the effectiveness of cortexin.

Received March 1, 2021

Accepted May 28, 2021

Maksimova Irina V. - assistant of the Department of Psychiatry and Narcology with a vocational education course, Krasnoyarsk State Medical University named after Professor V.F. Voyno-Yasenetsky, Krasnoyarsk, Russian Federation. Author ID RSCI 771740. ORCID iD 0000-0001-5076-5368. ResearcherID M-5458-2014. zabigylina@mail.ru

Berezovskaya Marina A., MD, associate professor, Head of the Department of Psychiatry and Narcology with the course of Postgraduate Education, Krasnoyarsk State Medical University named after Prof. V.F. Voino-Yasenetsky, Krasnoyarsk, Russian Federation. AuthorID 620131.

Berezovskaya Marina A., mberezovska@mail.ru 